\title{
Inelastic resonant tunneling through single molecules and quantum dots: spectrum modification due to nonequilibrium effects
}

\author{
D.A. Ryndyk* and J. Keller \\ Institut für Theoretische Physik, Universität Regensburg, Germany
}

(Dated: 6th July 2018)

\begin{abstract}
Resonant electron transport through a mesoscopic region (quantum dot or single molecule) with electron-phonon interaction is considered at finite voltage. In this case the standard LandauerBüttiker approach cannot be applied. Using the nonequilibrium Green function method we show that due to a nonequilibrium distribution function of electrons in the mesoscopic region, the inelastic scattering rate and spectral function of the dot become functions of the voltage and have to be calculated self-consistently.
\end{abstract}

During the past several years nonequilibrium quantum transport in nanostructures and, in particular, transport through mesoscopic tunneling structures, quantum dots (QD) and single molecules, is in the focus of both experimental and theoretical investigations because of possible electronic device applications. In this paper we reconsider one of the basic problems, namely resonant tunneling through a mesoscopic region with electron-phonon interaction, placed between macroscopic leads. We want to show that the nonequilibrium distribution function of electrons in the dot changes significantly the spectral function of the dot.

In the absence of interactions and in the limit of small voltage the current through a $\mathrm{QD}$ is described by the Landauer-Büttiker formula

$$
J=\frac{e}{2 \pi \hbar} \int T(\epsilon)\left[f^{0}\left(\epsilon-e \varphi_{L}\right)-f^{0}\left(\epsilon-e \varphi_{R}\right)\right] d \epsilon,
$$

where $T(\epsilon)$ is the transmission coefficient, $\varphi_{L(R)}$ is the electrical potentials of the left (right) lead. In the case of one resonant level with energy $\epsilon_{0}$, the transmission coefficient is given by the Breit-Wigner formula $T(\epsilon)=$ $\Gamma^{2} /\left[\left(\epsilon-\epsilon_{0}\right)^{2}+\Gamma^{2}\right]$, where $\Gamma$ is the level width produced by coupling to the leads. The corresponding spectral function of the dot, current-voltage curve and differential conductance are shown by the thin lines in Figs. 12

At finite voltage the current can be described by (1) with a voltage-independent transmission coefficient only in some special cases, e.g. for energy-independent coupling to the leads (wide-band limit). In the presence of interactions (e.g. internal Coulomb interaction and electron-phonon interaction ) $T(\epsilon)$ becomes temperature and voltage dependent.

The problem of inelastic resonant tunneling of electrons coupled to phonons was first considered in Refs. 1, 2, 3]. Phonon satellites are formed in the spectral function and in the differential conductance. Typical results are shown by dashed lines in Fig.1 1 and Fig. 2 Re-

*On leave from the Institute for Physics of Microstructures, RAS, Nizhny Novgorod, Russia cently this problem attracted attention after experiments on inelastic electron transport through single molecules [4, 5, 6, 7, 8, 9]. New theoretical treatments were presented in Refs. 10, 11, 12, 13, 14. In our paper we study the influence of the nonequilibrium electronic distribution function on the phonon self-energy. We find a significant shift and broadening of the phonon-satellites in the electronic spectral function. To our knowledge this effect has not been considered yet.

We use the Nonequilibrium Green Function (NGF) method [15, 16], which now is a standard approach in mesoscopic physics and molecular electronics 17, 18, 19, 20]. The advantage of the NGF formalism is that it can be successfully applied to a variety of systems and problems, that it is initially exact, and many powerful approximations can be derived from it (see [20, 21] and references therein).

We describe the electronic states of the mesoscopic region (QD) by a set a single-electron states $|\alpha\rangle$ with energies $\epsilon_{\alpha}$. These are coupled to the free condcuction electrons in the leads by the usual tunneling Hamiltonian. Furthermore the dot-electrons are coupled to vibrational modes. We do not consider Coulomb interaction in the dot, to avoid further complications, such as Coloumb blockade and Kondo effect. The system is then described by the following model Hamiltonian:

$$
H=H_{D}+H_{L}+H_{R}+H_{T}+H_{p h},
$$

with the dot-Hamiltonian

$$
H_{D}=\sum_{\alpha}\left(\epsilon_{\alpha}+e \varphi_{D}(t)\right) d_{\alpha}^{\dagger} d_{\alpha}
$$

the Hamiltonian of the right $(i=R)$ and left $(i=L)$ lead

$$
H_{i=L, R}=\sum_{k \sigma}\left(\epsilon_{i k \sigma}+e \varphi_{i}(t)\right) c_{i k \sigma}^{\dagger} c_{i k \sigma}
$$

and the tunneling Hamiltonian

$$
H_{T}=\sum_{i=L, R} \sum_{k \sigma, \alpha}\left(V_{i k \sigma, \alpha} c_{i k \sigma}^{\dagger} d_{\alpha}+\text { h.c. }\right) \text {. }
$$


Finally $H_{p h}$ describes phonons and electron-phonon coupling

$$
H_{e n v}^{p h}=\sum_{q} \hbar \omega_{q} a_{q}^{\dagger} a_{q}+\sum_{\alpha} \sum_{q} \lambda_{q \alpha}\left(a_{-q}+a_{q}^{\dagger}\right) d_{\alpha}^{\dagger} d_{\alpha},
$$

where $\hbar \omega_{q}$ is the phonon energy. The quantities $\varphi_{i}(t)$ and $\varphi_{D}(t)$ are the electrical potentials of the leads and the dot The latter will be set to zero in the following.

The current in direction from the left $(i=L)$ or right $(i=R)$ contact to the dot is found to be

$$
J_{i}(t)=\frac{2 e}{\hbar} \operatorname{Re}\left(\sum_{k \sigma, \alpha} V_{i k \sigma, \alpha} G_{\alpha, i k \sigma}^{<}(t, t)\right),
$$

where we define the lead-dot lesser Green function $G_{\alpha, i k \sigma}^{<}\left(t_{1}, t_{2}\right)=i\left\langle c_{i k \sigma}^{\dagger}\left(t_{2}\right) d_{\alpha}\left(t_{1}\right)\right\rangle$, while the charge of the $\mathrm{QD}$ is defined by the dot lesser function $G_{\alpha \beta}^{<}\left(t_{1}, t_{2}\right)=$ $i\left\langle d_{\beta}^{\dagger}\left(t_{2}\right) d_{\alpha}\left(t_{1}\right)\right\rangle$.

The equation of motion for the lesser Green function $G^{<}$contains coupling to the retarded and advanced Green functions $G^{R, A}$. In order to obtain a simple Dyson equation it is convenient to combine these functions to a matrix in Keldysh space 16, 20, 21]

$$
\breve{G}=\left(\begin{array}{cc}
G^{R} & G^{<} \\
0 & G^{A}
\end{array}\right) .
$$

where each component again is a matrix $G_{\eta, \eta^{\prime}}\left(t_{1}, t_{2}\right)$ within the states of the system including all the states of the leads $(\eta=i k \sigma)$ and the states of the $\operatorname{dot}(\eta=\alpha)$.

For this matrix Green function the nonequilibrium Dyson equation can be written in the usual way, in differential form

$$
\left[i \frac{\partial}{\partial t_{1}}-\breve{H}\left(t_{1}\right)\right] \breve{G}-\{\breve{\Sigma} \breve{G}\}=\breve{\delta},
$$

where $\breve{\delta}=\breve{I} \delta_{\eta \eta^{\prime}} \delta\left(t_{1}-t_{2}\right)$, and $\breve{I}$ is the unit matrix in Keldysh space. $\breve{H}(t)=\left(H_{L}+H_{R}+H_{D}+H_{T}\right) \breve{I}$ is the single-particle Hamiltonian. The curly brackets denote a matrix multiplication and convolution in time: $\{A B\}_{\eta \eta^{\prime}}\left(t_{1}, t_{2}\right)=\sum_{\gamma} \int d t_{3} A_{\eta \gamma}\left(t_{1}, t_{3}\right) B_{\gamma \eta^{\prime}}\left(t_{3}, t_{2}\right)$. The self-energy $\breve{\Sigma}$ describes interactions in the dot. The retarded and advanced Green functions and the spectral function $A=i\left(G^{R}-G^{A}\right)$ describe quasiparticle excitations. The lesser function $G^{<}$contains in addition a quantum distribution function.

In the absence of interactions in the leads (besides the tunneling) one can derive the following exact expression for the lead-dot function [17, 20]:

$$
\breve{G}_{\alpha, i k \sigma}=\sum_{\beta} V_{\beta, i k \sigma}\left\{\breve{G}_{\alpha \beta} \breve{G}_{i k \sigma}\right\}
$$

where $\breve{G}_{\alpha \beta}$ is the full dot Green function, while $\breve{G}_{i k \sigma}$ is the bare Green function of the leads, which is diagonal in the quantum numbers $i k \sigma$.

Substituting (10) in the general equation (9) we obtain the following basic equation for the dot Green function $\breve{\mathbf{G}}\left(t_{1}, t_{2}\right) \equiv \breve{G}_{\alpha \beta}\left(t_{1}, t_{2}\right)$ :

$$
\left(i \frac{\partial}{\partial t_{1}}-\mathbf{H}\right) \breve{\mathbf{G}}-\{\breve{\boldsymbol{\Sigma}} \breve{\mathbf{G}}\}=\breve{\mathbf{I}} \delta\left(t_{1}-t_{2}\right),
$$

with $\mathbf{H} \equiv H_{\alpha \beta}=\epsilon_{\alpha} \delta_{\alpha \beta}$. Here $\breve{\boldsymbol{\Sigma}}(\epsilon)=\breve{\boldsymbol{\Sigma}}_{L}^{(T)}(\epsilon)+\breve{\boldsymbol{\Sigma}}_{R}^{(T)}(\epsilon)+$ $\breve{\boldsymbol{\Sigma}}^{(p h)}(\epsilon)$ is the total self-energy of the dot composed of the tunneling self-energy

$$
\breve{\Sigma}_{j=L, R}^{(T)} \equiv \breve{\Sigma}_{j \alpha \beta}^{(T)}=\sum_{k \sigma}\left\{V_{j k \sigma, \alpha}^{*} \breve{G}_{j k \sigma} V_{j k \sigma, \beta}\right\} .
$$

and the self-energy $\breve{\boldsymbol{\Sigma}}^{(p h)} \equiv \breve{\Sigma}_{\alpha \beta}^{(p h)}$ describing interactions with phonons.

In the following we consider stationary transport produced by a time-independent voltage. In that case the Green functions depend only on the time difference. After Fourier transformation $\left(G(\epsilon)=\int G\left(t_{1}-t_{2}\right) e^{i \epsilon\left(t_{1}-t_{2}\right)} d\left(t_{1}-t_{2}\right)\right)$ one obtains

$$
(\epsilon \mathbf{I}-\mathbf{H}) \breve{\mathbf{G}}(\epsilon)-\breve{\mathbf{\Sigma}}(\epsilon) \breve{\mathbf{G}}(\epsilon)=\breve{\mathbf{I}},
$$
[17]

For the current we obtain the well-known expression

$$
\begin{aligned}
J_{i} & =\frac{i e}{\hbar} \int \frac{d \epsilon}{2 \pi} \operatorname{Tr}\left\{\boldsymbol { \Gamma } _ { i } ( \epsilon - e \varphi _ { i } ) \left(\mathbf{G}^{<}(\epsilon)+\right.\right. \\
& \left.\left.+f_{i}^{0}\left(\epsilon-e \varphi_{i}\right)\left[\mathbf{G}^{R}(\epsilon)-\mathbf{G}^{A}(\epsilon)\right]\right)\right\},
\end{aligned}
$$

with the level-width function

$$
\boldsymbol{\Gamma}_{i=L(R)}(\epsilon)=\Gamma_{i \alpha \beta}(\epsilon)=2 \pi \sum_{k \sigma} V_{i k \sigma, \beta} V_{i k \sigma, \alpha}^{*} \delta\left(\epsilon-\epsilon_{i k \sigma}\right) .
$$

Equations for the retarded and advanced functions follow from the diagonal part of (13)

$$
\left(\epsilon-\epsilon_{\alpha}\right) G_{\alpha \beta}^{R}-\sum_{\gamma} \Sigma_{\alpha \gamma}^{R} G_{\gamma \beta}^{R}=\delta_{\alpha \beta},
$$

and the equation for the lesser function (quantum kinetic equation) follows from the off-diagonal part of [13) combined with its conjugate $\breve{\mathbf{G}}(\epsilon)(\epsilon \mathbf{I}-\mathbf{H})-\breve{\mathbf{G}}(\epsilon) \breve{\mathbf{\Sigma}}(\epsilon)=\breve{\mathbf{I}}$ :

$$
\begin{gathered}
\left(\epsilon_{\beta}-\epsilon_{\alpha}\right) G_{\alpha \beta}^{<}-\sum_{\gamma}\left(\Sigma_{\alpha \gamma}^{R} G_{\gamma \beta}^{<}+\Sigma_{\alpha \gamma}^{<} G_{\gamma \beta}^{A}-\right. \\
\left.-G_{\alpha \gamma}^{R} \Sigma_{\gamma \beta}^{<}-G_{\alpha \gamma}^{<} \Sigma_{\gamma \beta}^{A}\right)=0 .
\end{gathered}
$$

In the following we will concentrate on the tunneling through one level $\alpha$ only. Then (16) simplifies further:

$$
\left(\Sigma_{\alpha}^{R}-\Sigma_{\alpha}^{A}\right) G_{\alpha}^{<}-\left(G_{\alpha}^{R}-G_{\alpha}^{A}\right) \Sigma_{\alpha}^{>}=0 .
$$


For the evaluation of the different terms in this equation we make the following ansatz for the lesser Green function of the dot

$$
\begin{gathered}
G_{\alpha}^{<}(\epsilon)=i A(\epsilon) f(\epsilon), \\
A(\epsilon)=i\left(G_{\alpha}^{R}-G_{\alpha}^{A}\right) .
\end{gathered}
$$

introducing a general nonequilibrium distribution function $f(\epsilon)$. For the Green functions of the leads in equilibrium we may use the Fermi function $f^{0}(\epsilon)$.

For the retarded tunneling self-energy $\Sigma^{R(T)}$ one obtains

$\Sigma_{i}^{R(T)}=\int \frac{d^{3} k}{(2 \pi)^{3}} \frac{\left|V_{i k, \alpha}\right|^{2}}{\epsilon-\epsilon_{k}-e \varphi_{i}+i 0}=\Lambda\left(\epsilon-e \varphi_{i}\right)-\frac{i}{2} \Gamma_{i}\left(\epsilon-e \varphi_{i}\right)$,

where $\Lambda$ is the real part of the self-energy, which usually can be included in the level energy $\epsilon_{\alpha}$, and $\Gamma$ describes level broadening due to coupling to the leads. For the corresponding lesser function one finds

$$
\Sigma_{i}^{<(T)}=i \Gamma_{i}\left(\epsilon-e \varphi_{i}\right) f^{0}\left(\epsilon-e \varphi_{i}\right) .
$$

In the following we will neglect the energy dependence of the level-width-function $\Gamma$. Then from (17) we obtain the following kinetic equation for the nonequilibrium distribution function $f(\epsilon)$ :

$$
\begin{gathered}
\Gamma_{L}\left[f(\epsilon)-f^{0}\left(\epsilon-e \varphi_{L}\right)\right]+\Gamma_{R}\left[f(\epsilon)-f^{0}\left(\epsilon-e \varphi_{R}\right)\right]+ \\
+i \Sigma^{<(p h)}(\epsilon)+i\left(\Sigma^{R(p h)}(\epsilon)-\Sigma^{A(p h)}(\epsilon)\right) f(\epsilon)=0 .
\end{gathered}
$$

Finally we have to evaluate the self-energy $\Sigma^{(p h)}$ due to the interaction with phonons. In order to demonstrate nonequilibrium effects most clearly we consider only one phonon mode with frequency $\omega_{0}$. In the standard selfconsistent Born approximation, using Keldysh technique, one obtains for the self-energies [20, 21]

$$
\begin{gathered}
\Sigma_{\epsilon}^{R(p h)}=\frac{i \lambda^{2}}{2} \int \frac{d \epsilon^{\prime}}{2 \pi}\left(G_{\epsilon-\epsilon^{\prime}}^{R} D_{\epsilon^{\prime}}^{K}+G_{\epsilon-\epsilon^{\prime}}^{K} D_{\epsilon^{\prime}}^{R}\right), \\
\Sigma_{\epsilon}^{A(p h)}=\frac{i \lambda^{2}}{2} \int \frac{d \epsilon^{\prime}}{2 \pi}\left(G_{\epsilon-\epsilon^{\prime}}^{A} D_{\epsilon^{\prime}}^{K}+G_{\epsilon-\epsilon^{\prime}}^{K} D_{\epsilon^{\prime}}^{A}\right), \\
\Sigma_{\epsilon}^{<(p h)}=i \lambda^{2} \int \frac{d \epsilon^{\prime}}{2 \pi} G_{\epsilon-\epsilon^{\prime}}^{<} D_{\epsilon^{\prime}}^{<.}
\end{gathered}
$$

where $G^{K}=2 G^{<}+G^{R}-G^{A}$ is the Keldysh Green function.

In the following we do not consider phonon renormalization and polaron effects, and use bare phonon Green functions:

$$
\begin{gathered}
D_{0}^{R}(\epsilon)=\frac{1}{\epsilon-\omega_{0}+i \delta}-\frac{1}{\epsilon+\omega_{0}+i \delta} \\
D_{0}^{<}(\epsilon)=-2 \pi i\left[\left(N_{0}+1\right) \delta\left(\epsilon+\omega_{0}\right)+N_{0} \delta\left(\epsilon-\omega_{0}\right)\right]
\end{gathered}
$$

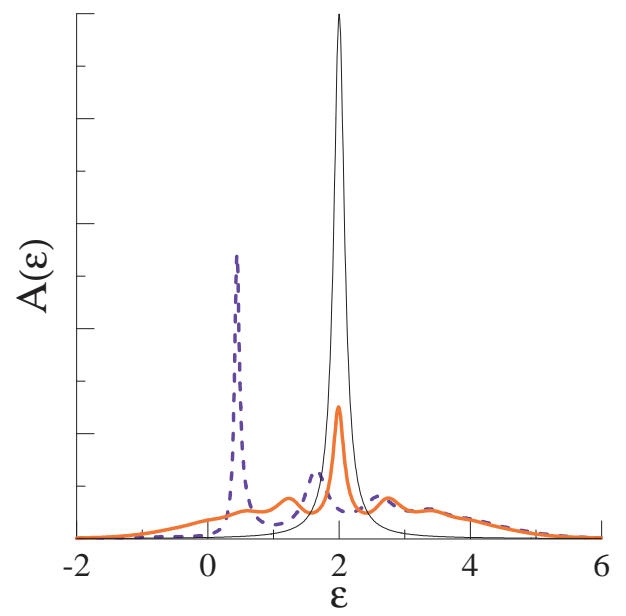

Figure 1: (Color online) Spectral function of the dot with energy level $\epsilon_{0}=2$. Thin line: no interactions, dashed line: interaction with phonon, electronic distribution in equilibrium, thick line: with phonon, including nonequilibrium effects at high voltage

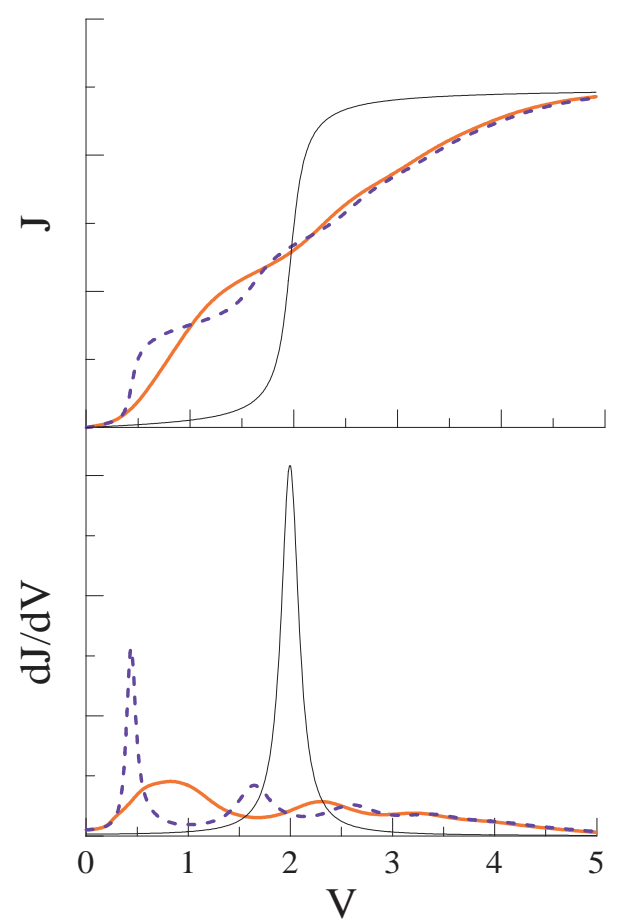

Figure 2: (Color online) Current-voltage curves and differential conductance $\left(\epsilon_{0}=2\right)$. For the legend of the curves see Fig. 1

where $N_{0}=1 /\left(e^{\omega_{0} / T}-1\right)$ is the equilibrium phonon population, (nonequilibrium effects in the phonon distribution was considered recently in 14]). However, we include nonequilibrium effects in the electron distribution function while calculating the self-energies. 


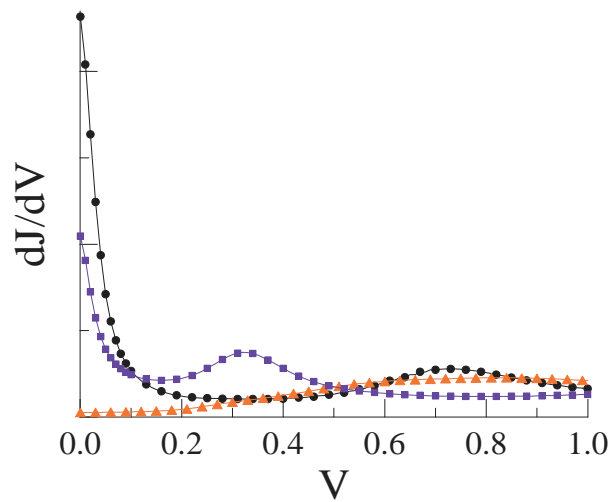

Figure 3: (Color online) Differential conductance at small voltages for different positions of the dot energy level $\epsilon_{0}=0$ (circles), $\epsilon_{0}=1$ (squares), and $\epsilon_{0}=2$ (triangles).

These self-energies enter the spectral function $A(\epsilon)=$ $i\left(G_{\alpha}^{R}(\epsilon)-G_{\alpha}^{A}(\epsilon)\right)$ of the quantum dot, where $G_{\alpha}^{R}(\epsilon)$ can be found from (15)

$$
G_{\alpha}^{R}(\epsilon)=\frac{1}{\epsilon-\epsilon_{\alpha}-\Sigma_{\epsilon}^{R(p h)}+i\left(\Gamma_{L}+\Gamma_{R}\right) / 2} .
$$

The real part of the self-energy leads to an energy shift in the spectral function, which depends on the applied voltage. To take this effect into account properly, we perform self-consistent calculations of the distribution function $f(\epsilon)$ (22) and self-energies (23)-(25) at finite voltage.

The spectral function is then used to calculate the current voltage curves. Combining $J_{L}$ and $J_{R}$ (see [17]) the expression for the current can be written as current

$J=\frac{e}{2 \pi \hbar} \frac{\Gamma_{L} \Gamma_{R}}{\Gamma_{R}+\Gamma_{L}} \int d \epsilon A(\epsilon)\left[f^{0}\left(\epsilon-e \varphi_{L}\right)-f^{0}\left(\epsilon-e \varphi_{R}\right)\right]$.

It looks as simple as the Landauer-Büttiker formula (1), but it is not trivial! The spectral density $A(\epsilon)$ now depends on the distribution function $f(\epsilon)$ and hence the applied voltage, $\varphi_{L}=-\varphi_{R}=V / 2$. Results for the current-voltage curves are presented in Fig.2 by thick lines.

If the spectral function $A(\epsilon)$ is calculated with help of the equilibrium distribution function $f^{0}(\epsilon)$, which does not depend on the voltage, the usual phonon satellites in the spectral function and differential conductance are obtained as shown by the dashed lines in Fig.11 and Fig.2 But if one takes into account the nonequilibrium distribution function $f(\epsilon)$, the spectral function changes with increasing voltage from the equilibrium one to the function shown as thick line in Fig.1 for a voltage $V>\epsilon_{0}$. Correspondingly, the phonon resonances in the differential conductance are shifted and suppressed (Fig.2] thick line).

Finally in Fig. 3 we show the differential conductance for different positions of the dot level $\epsilon_{0}$ with respect to the Fermi energy of the leads (defined in the absence of an applied voltage). For small dot energies the phonon peak appears at zero voltage and broadening is weak. This property can be used for experimental investigation of inelastic effects by tuning the dot level with help of a gate voltage.

In conclusion, we investigated inelastic resonant transport through a mesoscopic region. As an example, we considered resonant electron tunneling through a single level coupled to a single phonon mode. This model, although quite simple, is popular in connection with transport through vibrating molecules. The self-consistent treatment of the electronic nonequilibrium distribution function shows that the main peak and phonon sidebands in the differential conductance are shifted considerably and are essentially broadened as compared with their equilibrium position.

We thank K. Richter and G. Cuniberti for valuable discussions.

[1] L. I. Glazman and R. I. Shekhter, Zh. Eksp. Teor. Fiz. 94, 292 (1988), [Sov. Phys. JETP 67, 163 (1988)].

[2] N. S. Wingreen, K. W. Jacobsen, and J. W. Wilkins, Phys. Rev. Lett. 61, 1396 (1988); Phys. Rev. B 40, 11834 (1989).

[3] M. Jonson, Phys. Rev. B 39, 5924 (1989).

[4] M. A. Reed, et al., Science 278, 252 (1997).

[5] H. Park, et al., Nature 407, 57 (2000).

[6] J. Park, et al., Nature 417, 722 (2002).

[7] W. Liang, et al., Nature 417, 725 (2002).

[8] R. H. M. Smit, et al., Nature 419, 906 (2002).

[9] N. B. Zhitenev, H. Meng, and Z. Bao, Phys. Rev. Lett. 88, 226801 (2002).

[10] U. Lundin and R. H. McKenzie, Phys. Rev. B 66, 075303 (2002).

[11] J.-X. Zhu and A. V. Balatsky, Phys. Rev. B 67, 165326 (2003).

[12] S. Braig and K. Flensberg, Phys. Rev. B 68, 205324 (2003).

[13] V. Aji, J. E. Moore, and C. M. Varma, condmat/0302222.

[14] A. Mitra, I. Aleiner, and A. J. Millis, cond-mat/0311503.

[15] L. Kadanoff and G. Baym, Quantum Statistical Mechanics (Benjamin, New York, 1962).

[16] L. Keldysh, Zh. Eksp. Teor. Fiz. 47, 1515 (1964), [Sov. Phys. JETP 20, 1018 (1965)].

[17] Y. Meir and N. S. Wingreen, Phys. Rev. Lett. 68, 2512 (1992); A.-P. Jauho, N. S. Wingreen, and Y. Meir, Phys. Rev. B 50, 5528 (1994).

[18] E. Runge and H. Ehrenreich, Phys. Rev. B 45, 9145 (1992).

[19] S. Datta, Electronic Transport in Mesoscopic Systems (Cambridge University Press, Cambridge, 1995).

[20] H. Haug and A.-P. Jauho, Quantum Kinetics and Optics of Semiconductors, (Springer, 1996).

[21] J. Rammer and H. Smith, Rev. Mod. Phys. 58, 323 (1986). 